\title{
Homography-Based Visual Servo Tracking Control of a Wheeled Mobile Robot*
}

\author{
J. Chen ${ }^{\dagger}$, W. E. Dixon ${ }^{\ddagger}$, D. M. Dawson ${ }^{\dagger}$, and M. McIntire ${ }^{\dagger}$ \\ ${ }^{\dagger}$ Department of Electrical \& Computer Engineering, Clemson University, Clemson, SC 29634-0915 \\ ${ }^{\ddagger}$ Eng. Science and Tech. Div. - Robotics, Oak Ridge Nat. Lab., P.O. Box 2008, Oak Ridge, TN 37831-6305
}

E-mail: dixonwe@ornl.gov

\begin{abstract}
A visual servo tracking controller is developed in this paper for a monocular camera system mounted on an underactuated wheeled mobile robot (WMR) subject to nonholonomic motion constraints (i.e., the camera-in-hand problem). A prerecorded image sequence (e.g., a video) of three target points is used to define a desired trajectory for the WMR. By comparing the target points from the prerecorded sequence with the corresponding target points in the live image, projective geometric relationships are exploited to construct a Euclidean homography. The information obtained by decomposing the Euclidean homography is used to develop a kinematic controller. A Lyapunovbased analysis is used to develop an adaptive update law to actively compensate for the lack of depth information required for the translation error system.
\end{abstract}

\section{INTRODUCTION}

Wheeled mobile robots (WMRs) are often required to execute tasks in environments that are unstructured. Due to the uncertainty in the environment, an intelligent sensor that can enable autonomous navigation is well motivated. Given this motivation, researchers initially targeted the use of a variety of sonar and laser-based sensors. Some initial work also targeted the use of a fusion of various sensors to build a map of the environment for WMR navigation (see [17], [19], [28], [29], [31], and the references within). While this is still an active area of research, various shortcomings associated with these technologies and recent advances in image extraction/interpretation technology and advances in control theory have motivated researchers to investigate the sole use of camera-based vision systems for autonomous navigation. For example, using consecutive image frames and an object database, the authors of [18] recently proposed a monocular visual servo tracking controller for WMRs based on a linearized system of equations and Extended Kalman Filtering (EKF) techniques. Also using EKF techniques on the linearized kinematic model, the authors of [7] used feedback from a monocular omnidirectional camera system (similar to [1]) to enable

${ }^{*}$ This research was supported in part U.S. DOE Office of Biological and Environmental Research (OBER) Environmental Management Sciences Program (EMSP) project ID No. 82797 at ORNL for the DOE Office of Science (SC), a subcontract to ORNL by the Florida Department of Citrus through the University of Florida, and by U.S. NSF Grant DMI-9457967, ONR Grant N00014-99-1-0589, a DOC Grant, and an ARO Automotive Center Grant. wall following, follow-the-leader, and position regulation tasks. In [16], Hager et al. used a monocular vision system mounted on a pan-tilt-unit to generate image-Jacobian and geometry-based controllers by using different snapshots of the target and an epipolar constraint. As stated in [2], a drawback of the method developed in [16] is that the system equations became numerically ill-conditioned for large pan angles. Given this shortcoming, Burschka and Hager [2] used a spherical image projection of a monocular vision system that relied on teaching and replay phases to facilitate the estimation of the unknown object height parameter in the image-Jacobian by solving a least-squares problem. Spatiotemporal apparent velocities obtained from an optical flow of successive images of an object were used in [26] to estimate the depth and time-to-contact to develop a monocular vision guide robot. A similar optical flow technique was also used in [20]. In [9], Dixon et al. used feedback from an uncalibrated, fixed (ceiling-mounted) camera to develop an adaptive tracking controller for a WMR that compensated for the parametric uncertainty in the camera and the WMR dynamics. An image-based visual servo controller that exploits an object model was proposed in [30] to solve the WMR tracking controller (the regulation problem was not solved due to restrictions on the reference trajectory) that adapted for the constant, unknown height of an object moving in a plane through Lyapunov-based techniques. In [21] and [33], visual servo controllers were recently developed for systems with similar underactuated kinematics as WMRs. Specifically, Mahony and Hamel [21] developed a semi-global asymptotic visual servoing result for unmanned aerial vehicles that tracked parallel coplanar linear visual features while Zhang and Ostrowski [33] used a vision system to navigate a blimp.

In contrast to the previous image-based visual servo control approaches, novel homography-based visual servo control techniques have been recently developed in a series of papers by Malis and Chaumette (e.g., [3], [4], [22], [23], $[24])$. The homography-based approach exploits a combination of reconstructed Euclidean information and imagespace information in the control design. The Euclidean information is reconstructed by decoupling the interaction between translation and rotation components of a homography matrix. As stated in [24], some advantages of this methodology over the aforementioned approaches are that an accurate Euclidean model of the environment (or target image) is not required and potential singularities in the 
image-Jacobian are eliminated (i.e., the image-Jacobian for homography-based visual servo controllers is typically triangular). Motivated by the advantages of the homographybased strategy, several researchers have recently developed various regulation controllers for robot manipulators (see [5], [6], [8], [11], and [13]). In [12], a homography-based visual servo control strategy was recently developed to asymptotically regulate the position/orientation of a WMR to a constant Euclidean position defined by a reference image, despite unknown depth information.

In this paper, a homography-based visual servo control strategy is used to force the Euclidean position/orientation of a camera mounted on a WMR (i.e., the camera in hand problem) to track a desired time-varying trajectory defined by a prerecorded sequence of images. By comparing the features of an object from a reference image to features of an object in the current image and the prerecorded sequence of images, projective geometric relationships are exploited to enable the reconstruction of the Euclidean coordinates of the target points with respect to the WMR coordinate frame. The tracking control objective is naturally defined in terms of the Euclidean space, however, the translation error is unmeasurable. That is, the Euclidean reconstruction is scaled by an unknown distance from the camera/WMR to the target, and while the scaled position is measurable through the homography, the unscaled position error is unmeasurable. To overcome this obstacle, a Lyapunov-based control strategy is employed that provides a framework for the construction of an adaptive update law to actively compensate for the unknown depth-related scaling constant. While similar techniques as in [12] are employed for the Euclidean reconstruction from the image data for the WMR system, new development is provided in this paper to develop a tracking controller. In contrast to visual servo methods that linearize the system equations to facilitate EKF methods, the Lyapunov-based control design in this paper is based on the full nonlinear kinematic model of the vision system and the mobile robot system.

\section{Problem Formulation}

As illustrated in Fig. 1, the origin of the orthogonal coordinate system $\mathcal{F}$ attached to the camera is coincident with the center of the WMR wheel axis (i.e., the camera is "in-hand"). As also illustrated in Fig. 1, the $x y$-axis of $\mathcal{F}$ defines the plane of motion where the $x$-axis of $\mathcal{F}$ is perpendicular to the wheel axis, and the $y$-axis is parallel to the wheel axis. The $z$-axis of $\mathcal{F}$ is perpendicular to the plane of motion and is located at the center of the wheel axis. The linear velocity of the WMR along the $x$-axis is denoted by $v_{c}(t) \in \mathbb{R}$, and the angular velocity $\omega_{c}(t) \in \mathbb{R}$ is about the T-axis (see Fig. 1). The desired trajectory is defined by the prerecorded time-varying trajectory of $\mathcal{F}_{d}$ that is assumed to be second-order differentiable. The desired trajectory is obtained from a prerecorded set of images of a stationary target viewed by the on-board camera as the WMR moves. For example, the desired WMR motion could be obtained as an operator drives the robot via a teach pendant, and the on-board camera captures and stores the sequence of images of the stationary target. A fixed orthogonal coordinate system, denoted by $\mathcal{F}^{*}$, represents a fixed (i.e., a single snapshot) reference position and orientation of the camera relative to the stationary target. Based on the definition of these coordinate frames, the goal of this paper is to develop a homography-based visual servo controller that will force $\mathcal{F}$ to track the position and orientation trajectory provided by $\mathcal{F}_{d}$.

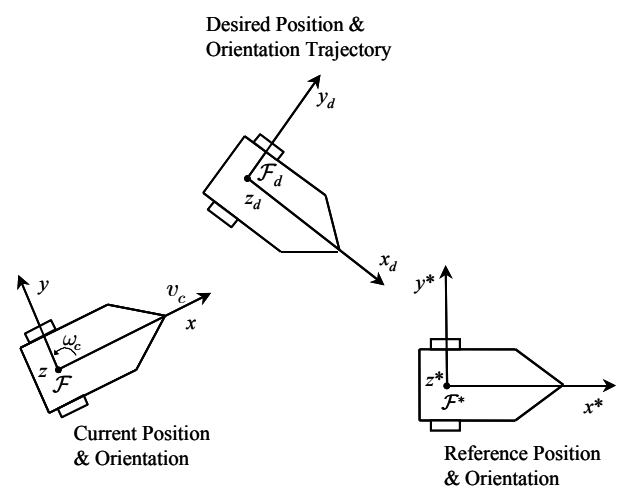

Fig. 1. Mobile robot coordinate systems.

\section{A. Geometric Model}

In this section, geometric relationships are developed between the coordinate systems $\mathcal{F}, \mathcal{F}_{d}$, and $\mathcal{F}^{*}$, and a reference plane $\pi$ that is defined by three target points $O_{i}$ $\forall i=1,2,3$ that are not collinear. The 3D Euclidean coordinates of $O_{i}$ expressed in terms of $\mathcal{F}, \mathcal{F}_{d}$, and $\mathcal{F}^{*}$ as $\bar{m}_{i}(t)$, $\bar{m}_{d i}(t), \bar{m}_{i}^{*} \in \mathbb{R}^{3}$, respectively, are defined as follows (see Fig. 2)

$$
\begin{aligned}
\bar{m}_{i}(t) & \triangleq\left[\begin{array}{lll}
x_{i}(t) & y_{i}(t) & z_{i}(t)
\end{array}\right]^{T} \\
\bar{m}_{d i}(t) & \triangleq\left[\begin{array}{lll}
x_{d i}(t) & y_{d i}(t) & z_{d i}(t)
\end{array}\right]^{T} \\
\bar{m}_{i}^{*} & \triangleq\left[\begin{array}{lll}
x_{i}^{*} & y_{i}^{*} & z_{i}^{*}
\end{array}\right]^{T}
\end{aligned}
$$

under the standard assumption that the distances from the origin of the respective coordinate frames to the targets along the focal axis remains positive (i.e., $x_{i}(t), x_{d i}(t), x_{i}^{*} \geq \varepsilon>0$ where $\varepsilon$ is an arbitrarily small positive constant). The rotation from $\mathcal{F}^{*}$ to $\mathcal{F}$ is denoted by $R(t) \in S O(3)$, and the translation from $\mathcal{F}$ to $\mathcal{F}^{*}$ is denoted by $x_{f}(t) \in \mathbb{R}^{3}$ where $x_{f}(t)$ is expressed in $\mathcal{F}$. Similarly, $R_{d}(t) \in S O(3)$ denotes the desired time-varying rotation from $\mathcal{F}^{*}$ to $\mathcal{F}_{d}$, and $x_{f d}(t) \in \mathbb{R}^{3}$ denotes the desired translation from $\mathcal{F}_{d}$ to $\mathcal{F}^{*}$ where $x_{f d}(t)$ is expressed in $\mathcal{F}_{d}$. Since the motion of the WMR is constrained to a plane, $x_{f}(t)$ and $x_{f d}(t)$ are defined as follows

$$
\begin{aligned}
x_{f}(t) & \triangleq\left[\begin{array}{lll}
x_{f 1} & x_{f 2} & 0
\end{array}\right]^{T} \\
x_{f d}(t) & \triangleq\left[\begin{array}{lll}
x_{f d 1} & x_{f d 2} & 0
\end{array}\right]^{T} .
\end{aligned}
$$

From the geometry between the coordinate frames depicted in Fig. 2, $\bar{m}_{i}^{*}$ can be related to $\bar{m}_{i}(t)$ and $\bar{m}_{d i}(t)$ as follows

$$
\bar{m}_{i}=x_{f}+R \bar{m}_{i}^{*} \quad \bar{m}_{d i}=x_{f d}+R_{d} \bar{m}_{i}^{*} .
$$


In (3), $R(t)$ and $R_{d}(t)$ are defined as follows

$$
\begin{aligned}
& R \triangleq[ {\left[\begin{array}{lll}
\cos \theta & -\sin \theta & 0 \\
\sin \theta & \cos \theta & 0 \\
0 & 0 & 1
\end{array}\right], } \\
& R_{d} \triangleq\left[\begin{array}{lll}
\cos \theta_{d} & -\sin \theta_{d} & 0 \\
\sin \theta_{d} & \cos \theta_{d} & 0 \\
0 & 0 & 1
\end{array}\right]
\end{aligned}
$$

where $\theta(t) \in \mathbb{R}$ denotes the right-handed rotation angle about $z_{i}(t)$ that aligns the rotation of $\mathcal{F}$ with $\mathcal{F}^{*}$, and $\theta_{d}(t) \in \mathbb{R}$ denotes the right-handed rotation angle about $z_{d i}(t)$ that aligns the rotation of $\mathcal{F}_{d}$ with $\mathcal{F}^{*}$. From Fig. 1 and the definitions of $\theta(t)$ and $\theta_{d}(t)$, it is clear that

$$
\dot{\theta}=-\omega_{c} \quad \dot{\theta}_{d}=-\omega_{c d} .
$$

The rotation angles are assumed to be confined to the following regions

$$
-\pi<\theta(t)<\pi \quad-\pi<\theta_{d}(t)<\pi .
$$

From the geometry given in Fig. 2, the distance $d^{*} \in \mathbb{R}$ from $\mathcal{F}^{*}$ to $\pi$ along the unit normal is given by

$$
d^{*}=n^{* T} \bar{m}_{i}^{*}
$$

where $n^{*}=\left[\begin{array}{lll}n_{x}^{*} & n_{y}^{*} & n_{z}^{*}\end{array}\right]^{T} \in \mathbb{R}^{3}$ denotes the constant unit normal to $\pi$. From (7), the relationships in (3) can be expressed as follows

$$
\bar{m}_{i}=\left(R+\frac{x_{f}}{d^{*}} n^{* T}\right) \bar{m}_{i}^{*} \quad \bar{m}_{d i}=\left(R_{d}+\frac{x_{f d}}{d^{*}} n^{* T}\right) \bar{m}_{i}^{*} .
$$

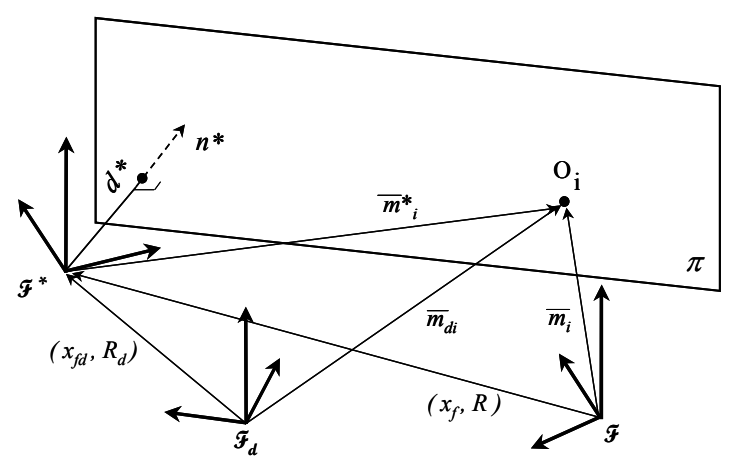

Fig. 2. Coordinate frame relationships.

\section{B. Euclidean Reconstruction}

The relationship given in (3) provides a means to quantify the translational and rotational error between $\mathcal{F}$ and $\mathcal{F}^{*}$ and between $\mathcal{F}_{d}$ and $\mathcal{F}^{*}$. Since the position of $\mathcal{F}$, $\mathcal{F}_{d}$, and $\mathcal{F}^{*}$ cannot be directly measured, this section illustrates how the normalized Euclidean coordinates of the target points can be reconstructed by relating multiple images. Specifically, comparisons are made between an image acquired from the camera attached to $\mathcal{F}$, the reference image, and the prerecorded sequence of images that define the trajectory of $\mathcal{F}_{d}$. To facilitate the subsequent development, the normalized Euclidean coordinates of $O_{i}$ expressed in terms of $\mathcal{F}, \mathcal{F}_{d}$, and $\mathcal{F}^{*}$ as $m_{i}(t), m_{d i}(t), m_{i}^{*} \in \mathbb{R}^{3}$, respectively, are defined as follows

$$
\begin{aligned}
& m_{i} \triangleq\left[\begin{array}{lll}
1 & m_{i y} & m_{i z}
\end{array}\right]^{T}=\frac{\bar{m}_{i}}{x_{i}} \\
& m_{d i} \triangleq\left[\begin{array}{lll}
1 & m_{d i y} & m_{d i z}
\end{array}\right]^{T}=\frac{\bar{m}_{d i}}{x_{d i}} \\
& m_{i}^{*} \triangleq\left[\begin{array}{lll}
1 & m_{i y}^{*} & m_{i z}^{*}
\end{array}\right]^{T}=\frac{\bar{m}_{i}^{*}}{x_{i}^{*}}
\end{aligned}
$$

where $\bar{m}_{i}(t), \bar{m}_{d i}(t)$, and $\bar{m}_{i}^{*}$ are introduced in (1). In addition to having a Euclidean coordinate, each target point $O_{i}$ will also have a projected pixel coordinate denoted by $u_{i}(t), v_{i}(t) \in \mathbb{R}$ for $\mathcal{F}, u_{i}^{*}, v_{i}^{*} \in \mathbb{R}$ for $\mathcal{F}^{*}$, and $u_{d i}(t), v_{d i}(t) \in \mathbb{R}$ for $\mathcal{F}_{d}$, that are defined as elements of $p_{i}(t) \in \mathbb{R}^{3}$ (i.e., the actual time-varying image points), $p_{d i}(t) \in \mathbb{R}^{3}$ (i.e., the desired image point trajectory), and $p_{i}^{*} \in \mathbb{R}^{3}$ (i.e., the constant reference image points), respectively, as follows

$$
\begin{gathered}
p_{i} \triangleq\left[\begin{array}{lll}
1 & v_{i} & u_{i}
\end{array}\right]^{T} \quad p_{d i} \triangleq\left[\begin{array}{lll}
1 & v_{d i} & u_{d i}
\end{array}\right]^{T} \\
p_{i}^{*} \triangleq\left[\begin{array}{lll}
1 & v_{i}^{*} & u_{i}^{*}
\end{array}\right]^{T} .
\end{gathered}
$$

The normalized Euclidean coordinates of the target points are related to the image data through the following pinhole lens models

$$
p_{i}=A m_{i} \quad p_{d i}=A m_{d i} \quad p_{i}^{*}=A m_{i}^{*}
$$

where $A \in \mathbb{R}^{3 \times 3}$ is a known, constant, and invertible intrinsic camera calibration matrix.

Given that $m_{i}(t), m_{d i}(t)$, and $m_{i}^{*}$ can be obtained from (11), the rotation and translation between the coordinate systems can now be related in terms of the normalized Euclidean coordinates as follows

$$
\begin{aligned}
& m_{i}=\underbrace{\frac{x_{i}^{*}}{x_{i}}}_{\alpha_{i}} \underbrace{\left(R+x_{h} n^{* T}\right)}_{H} m_{i}^{*} \\
& m_{d i}=\underbrace{\frac{x_{i}^{*}}{x_{d i}}}_{\alpha_{d i}} \underbrace{\left(R_{d}+x_{h d} n^{* T}\right)}_{H_{d}} m_{i}^{*}
\end{aligned}
$$

where $\alpha_{i}(t), \alpha_{d i}(t) \in \mathbb{R}$ denote the depth ratios, $H(t)$, $H_{d}(t) \in \mathbb{R}^{3 \times 3}$ denote Euclidean homographies, and $x_{h}(t)$, $x_{h d}(t) \in \mathbb{R}^{3}$ denote scaled translation vectors that are defined as follows

$$
\begin{aligned}
x_{h} & \triangleq\left[\begin{array}{lll}
x_{h 1} & x_{h 2} & 0
\end{array}\right]^{T}=\frac{x_{f}}{d^{*}} \\
x_{h d} & \triangleq\left[\begin{array}{lll}
x_{h d 1} & x_{h d 2} & 0
\end{array}\right]^{T}=\frac{x_{f d}}{d^{*}} .
\end{aligned}
$$

By using (4) and (14), the Euclidean homography in (12) can be rewritten as follows

$$
H=\left[\begin{array}{ccc}
\cos \theta+x_{h 1} n_{x}^{*} & -\sin \theta+x_{h 1} n_{y}^{*} & x_{h 1} n_{z}^{*} \\
\sin \theta+x_{h 2} n_{x}^{*} & \cos \theta+x_{h 2} n_{y}^{*} & x_{h 2} n_{z}^{*} \\
0 & 0 & 1
\end{array}\right]
$$


By examining the terms in (15), it is clear that $H(t)$ contains signals that are not directly measurable (e.g., $\theta(t)$, $x_{h}(t)$, and $\left.n^{*}\right)$. By expanding $H_{j k}(t) \forall j=1,2, k=1,2,3$, the following expressions can be obtained from (9), (12), and (15)

$$
\begin{aligned}
1 & =\alpha_{i}\left(H_{11}+H_{12} m_{i y}^{*}+H_{13} m_{i z}^{*}\right) \\
m_{i y} & =\alpha_{i}\left(H_{21}+H_{22} m_{i y}^{*}+H_{23} m_{i z}^{*}\right) \\
m_{i z} & =\alpha_{i} m_{i z}^{*} .
\end{aligned}
$$

From (16)-(18), it is clear that three independent equations with nine unknowns (i.e., $H_{j k}(t) \forall j=1,2, k=1,2,3$ and $\left.\alpha_{i}(t) \forall i=1,2,3\right)$ can be generated for each target point. Hence, by determining the normalized Euclidean coordinate of three target points in $\mathcal{F}$ and $\mathcal{F}^{*}$ from the image data and (11), the unknown elements of $H(t)$ and the unknown ratio $\alpha_{i}(t)$ can be determined. Likewise, for the same three target points in $\mathcal{F}_{d}$ and $\mathcal{F}^{*}$, the unknown elements of $H_{d}(t)$ and the unknown ratio $\alpha_{d i}(t)$ can be determined. Once the elements of $H(t)$ and $H_{d}(t)$ are determined, various techniques (e.g., see [15], [32]) can be used to decompose the Euclidean homographies to obtain the rotation and translation components. Hence, $R(t), R_{d}(t)$ $x_{h}(t)$, and $x_{h d}(t)$ are all known signals that can be used for the subsequent control synthesis. Since $R(t)$ and $R_{d}(t)$ are known matrices, then (4) can be used to determine $\theta(t)$ and $\theta_{d}(t)$.

Remark 1: To develop a tracking controller, it is typical that the desired trajectory is used as a feedforward component in the control design. Hence, for a kinematic controller the desired trajectory is required to be at least first order differentiable and at least second order differentiable for a dynamic level controller. From the Euclidean homography introduced in $(13), m_{d}(t)$ can be expressed in terms of the a priori known, functions $\alpha_{d i}(t), H_{d}(t), R_{d}(t)$, and $x_{h d}(t)$. Since these signals can be obtained from the prerecorded sequence of images, sufficiently smooth functions can be generated for these signals by fitting a sufficiently smooth spline function to the signals. Hence, in practice, the a priori developed smooth functions $\alpha_{d i}(t), R_{d}(t)$, and $x_{h d}(t)$ can be constructed as bounded functions with sufficiently bounded time derivatives. Given $\theta_{d}(t)$ and the time derivative of $R_{d}(t), \dot{\theta}_{d}(t)$ can be determined. In the subsequent tracking control development, $\dot{x}_{h d 1}(t)$ and $\dot{\theta}_{d}(t)$ will be used as feedforward control terms.

\section{Control Development}

The control objective is to ensure that the coordinate frame $\mathcal{F}$ tracks the time-varying trajectory of $\mathcal{F}_{d}$ (i.e., $\bar{m}_{i}(t)$ tracks $\left.\bar{m}_{d i}(t)\right)$. This objective is naturally defined in terms of the Euclidean position/orientation of the WMR. Specifically, based on the previous development, the translation and rotation tracking error, denoted by $e(t) \triangleq$ $\left[\begin{array}{lll}e_{1} & e_{2} & e_{3}\end{array}\right]^{T} \in \mathbb{R}^{3}$, is defined as follows

$$
\begin{aligned}
& e_{1} \triangleq x_{h 1}-x_{h d 1} \\
& e_{2} \triangleq x_{h 2}-x_{h d 2} \\
& e_{3} \triangleq \theta-\theta_{d}
\end{aligned}
$$

where $x_{h 1}(t), x_{h 2}(t), x_{h d 1}(t)$, and $x_{h d 2}(t)$ are introduced in $(14)$, and $\theta(t)$ and $\theta_{d}(t)$ are introduced in (4). Based on the definition in (19), it can be shown that the control objective is achieved if the tracking error $e(t) \rightarrow 0$. Specifically, it is clear from (14) that if $e_{1}(t) \rightarrow 0$ and $e_{2}(t) \rightarrow 0$, then $x_{f}(t) \rightarrow x_{f d}(t)$. If $e_{3} \rightarrow 0$, then it is clear from (4) and (19) that $R(t) \rightarrow R_{d}(t)$. If $x_{f}(t) \rightarrow x_{f d}(t)$ and $R(t) \rightarrow R_{d}(t)$, then (3) can be used to prove that $\bar{m}_{i}(t) \rightarrow \bar{m}_{d i}(t)$.

\section{A. Open-loop Error System}

As a means to develop the open-loop tracking error system, the time derivative of the Euclidean position $x_{f}(t)$ is determined as follows [24]

$$
\dot{x}_{f}=-v+\left[x_{f}\right]_{\times} \omega
$$

where $v(t), \omega(t) \in \mathbb{R}^{3}$ denote the respective linear and angular velocity of the WMR expressed in $\mathcal{F}$ as

$$
v \triangleq\left[\begin{array}{lll}
v_{c} & 0 & 0
\end{array}\right]^{T} \quad \omega \triangleq\left[\begin{array}{lll}
0 & 0 & \omega_{c}
\end{array}\right]^{T},
$$

and $\left[x_{f}\right]_{\times}$denotes the $3 \times 3$ skew-symmetric form of $x_{f}(t)$. After substituting (14) into (20), the time derivative of the translation vector $x_{h}(t)$ can be written in terms of the linear and angular velocity of the WMR as follows

$$
\dot{x}_{h}=-\frac{v}{d^{*}}+\left[x_{h}\right]_{\times} \omega .
$$

After incorporating (21) into (22), the following expression can be obtained

$$
\begin{aligned}
& \dot{x}_{h 1}=-\frac{v_{c}}{d^{*}}+x_{h 2} \omega_{c} \\
& \dot{x}_{h 2}=-x_{h 1} \omega_{c}
\end{aligned}
$$

where (14) was utilized. Given that the desired trajectory is generated from a prerecorded set of images taken by the on-board camera as the WMR was moving, a similar expression as (22) can be developed as follows

$$
\dot{x}_{f d}=-\left[\begin{array}{lll}
v_{c d} & 0 & 0
\end{array}\right]^{T}+\left[x_{f d}\right]_{\times}\left[\begin{array}{lll}
0 & 0 & \omega_{c d}
\end{array}\right]^{T}
$$

where $v_{c d}(t), \omega_{c d}(t) \in \mathbb{R}$ denote the respective desired linear ${ }^{1}$ and angular velocity of the WMR expressed in $\mathcal{F}_{d}$. After substituting (14) into (24), the time derivative of the translation vector $x_{h d}(t)$ can be written as follows

$$
\begin{aligned}
& \dot{x}_{h d 1}=-\frac{v_{c d}}{d^{*}}+x_{h d 2} \omega_{c d} \\
& \dot{x}_{h d 2}=-x_{h d 1} \omega_{c d}
\end{aligned}
$$

where (14) was utilized. After taking the time derivative of (19) and utilizing (5) and (23), the following open-loop error system can be obtained

$$
\begin{aligned}
& d^{*} \dot{e}_{1}=-v_{c}+d^{*}\left(x_{h 2} \omega_{c}-\dot{x}_{h d 1}\right) \\
& \dot{e}_{2}=-\left(x_{h 1} \omega_{c}+x_{h d 1} \dot{\theta}_{d}\right) \\
& \dot{e}_{3}=-\left(\omega_{c}+\dot{\theta}_{d}\right)
\end{aligned}
$$

${ }^{1}$ Note that $v_{c d}(t)$ is not measurable. 
where the definition of $e_{2}(t)$ given in (19), and the second equation of (25) was utilized. To facilitate the subsequent development, the auxiliary variable $\bar{e}_{2}(t) \in \mathbb{R}$ is defined as

$$
\bar{e}_{2} \triangleq e_{2}-x_{h d 1} e_{3} .
$$

After taking the time derivative of (27) and utilizing (26), the following expression is obtained

$$
\dot{\bar{e}}_{2}=-\left(e_{1} \omega_{c}+\dot{x}_{h d 1} e_{3}\right) .
$$

Based on (27), it is clear that if $\bar{e}_{2}(t), e_{3}(t) \rightarrow 0$, then $e_{2}(t) \rightarrow 0$. Based on this observation and the open-loop dynamics given in (28), the following control development is based on the desire to prove that $e_{1}(t), \bar{e}_{2}(t), e_{3}(t)$ are asymptotically driven to zero.

\section{B. Closed-Loop Error System}

Based on the open-loop error systems in (26) and (28), the linear and angular velocity kinematic control inputs for the WMR are designed as follows

$$
\begin{gathered}
v_{c} \triangleq k_{v} e_{1}-\bar{e}_{2} \omega_{c}+\hat{d}^{*}\left(x_{h 2} \omega_{c}-\dot{x}_{h d 1}\right) \\
\omega_{c} \triangleq k_{\omega} e_{3}-\dot{\theta}_{d}-\dot{x}_{h d 1} \bar{e}_{2}
\end{gathered}
$$

where $k_{v}, k_{\omega} \in \mathbb{R}$ denote positive, constant control gains. In (29), the parameter update law $\hat{d}^{*}(t) \in \mathbb{R}$ is generated by the following differential equation

$$
\dot{\hat{d}}^{*} \triangleq \gamma_{1} e_{1}\left(x_{h 2} \omega_{c}-\dot{x}_{h d 1}\right)
$$

where $\gamma_{1} \in \mathbb{R}$ is a positive, constant adaptation gain. After substituting the kinematic control signals designed in (29) and (30) into (26), the following closed-loop error systems are obtained

$$
\begin{aligned}
& d^{*} \dot{e}_{1}=-k_{v} e_{1}+\bar{e}_{2} \omega_{c}+\tilde{d}^{*}\left(x_{h 2} \omega_{c}-\dot{x}_{h d 1}\right) \\
& \dot{\bar{e}}_{2}=-\left(e_{1} \omega_{c}+\dot{x}_{h d 1} e_{3}\right) \\
& \dot{e}_{3}=-k_{\omega} e_{3}+\dot{x}_{h d 1} \bar{e}_{2}
\end{aligned}
$$

where (28) was utilized and the depth-related parameter estimation error $\tilde{d}^{*}(t) \in \mathbb{R}$ is defined as follows

$$
\tilde{d}^{*} \triangleq d^{*}-\hat{d}^{*}
$$

\section{Stability Analysis}

Theorem 1: The adaptive update law defined in (31) along with the control input designed in (29) and (30) ensure that the WMR tracking error $e(t)$ is asymptotically driven to zero in the sense that

$$
\lim _{t \rightarrow \infty} e(t)=0
$$

provided the time derivative of the desired trajectory satisfies the following condition

$$
\lim _{t \rightarrow \infty} \dot{x}_{h d 1} \neq 0
$$

Proof: To prove Theorem 1, the non-negative function $V(t) \in \mathbb{R}$ is defined as follows

$$
V \triangleq \frac{1}{2} d^{*} e_{1}^{2}+\frac{1}{2} \bar{e}_{2}^{2}+\frac{1}{2} e_{3}^{2}+\frac{1}{2 \gamma_{1}} \tilde{d}^{* 2} .
$$

The following simplified expression can be obtained by taking the time derivative of (36), substituting the closed-loop dynamics in (32) into the resulting expression, and then cancelling common terms

$$
\dot{V}=-k_{v} e_{1}^{2}+e_{1} \tilde{d}^{*}\left(x_{h 2} \omega_{c}-\dot{x}_{h d 1}\right)-k_{\omega} e_{3}^{2}-\frac{1}{\gamma_{1}} \tilde{d}^{*} \dot{\hat{d}}^{*} .
$$

After substituting (31) into (37), the following expression can be obtained

$$
\dot{V}=-k_{v} e_{1}^{2}-k_{\omega} e_{3}^{2}
$$

From (36) and (38), it is clear that $e_{1}(t), \bar{e}_{2^{2}}(t), e_{3}(t)$, $\tilde{d}^{*}(t) \in \mathcal{L}_{\infty}$ and that $e_{1}(t), e_{3}(t) \in \mathcal{L}_{\in}$. Since $\tilde{d}^{*}(t) \in \mathcal{L}_{\infty}$ and $d^{*}$ is a constant, the expression in (33) can be used to determine that $\hat{d}^{*}(t) \in \mathcal{L}_{\infty}$. From the assumption that $x_{h d 1}(t), \dot{x}_{h d 1}(t), x_{h d 2}(t), \theta_{d}(t)$, and $\dot{\theta}_{d}(t)$ are constructed as bounded functions, and the fact that $\bar{e}_{2}(t), e_{3}(t) \in \mathcal{L}_{\infty}$, the expressions in (19), (27), and (30) can be used to prove that $e_{2}(t), x_{h 1}(t), x_{h 2}(t), \theta(t), \omega_{c}(t) \in \mathcal{L}_{\infty}$. Based on the previous development, the expressions in (29), (31), and (32) can be used to conclude that $v_{c}(t), \dot{\hat{d}}^{*}(t), \dot{e}_{1}(t), \dot{\bar{e}}_{2}(t)$, $\dot{e}_{3}(t) \in \mathcal{L}_{\infty}$. Based on the fact that $e_{1}(t), e_{3}(t), \dot{e}_{1}(t)$, $\dot{e}_{3}(t) \in \mathcal{L}_{\infty}$ and that $e_{1}(t), e_{3}(t) \in \mathcal{L}_{\in}$, Barbalat's lemma [25] can be employed to prove that

$$
\lim _{t \rightarrow \infty} e_{1}(t), e_{3}(t)=0 .
$$

From (39) and the fact that the signal $\dot{x}_{h d 1}(t) \bar{e}_{2}(t)$ is uniformly continuous (i.e., $\dot{x}_{h d 1}(t), \ddot{x}_{h d 1}(t), \bar{e}_{2}(t), \dot{\bar{e}}_{2}(t) \in$ $\left.\mathcal{L}_{\infty}\right)$, the Extended Barbalat's Lemma [10] can be applied to the last equation in (32) to prove that

$$
\lim _{t \rightarrow \infty} \dot{e}_{3}(t)=0
$$

and that

$$
\lim _{t \rightarrow \infty} \dot{x}_{h d 1}(t) \bar{e}_{2}(t)=0 .
$$

If the desired trajectory satisfies (35), then (41) can be used to prove that

$$
\lim _{t \rightarrow \infty} \bar{e}_{2}(t)=0 .
$$

Based on the definition of $\bar{e}_{2}(t)$ given in (27), the results in (39) and (42) can be used to conclude that

$$
\lim _{t \rightarrow \infty} e_{2}(t)=0
$$

provided the condition in (35) is satisfied.

Remark 2: The condition given in (35) is in terms of the time derivative of the desired translation vector. Typically, for WMR tracking problems, this assumption is expressed in terms of the desired linear and angular velocity of the WMR. To this end, (25) can be substituted into (35) to obtain the following condition

$$
\lim _{t \rightarrow \infty} \frac{v_{c d}(t)}{d^{*}} \neq x_{h d 2}(t) \omega_{c d}(t) .
$$

The condition in (44) is comparable to typical WMR tracking results that restrict the desired linear and angular velocity. For an in-depth discussion of this type of restriction including related previous results see [10]. 


\section{Conclusions}

In this paper, the position/orientation of a WMR is forced to track a desired time-varying trajectory defined by a prerecorded sequence of images. To achieve the result, multiple views of three target points were used to develop Euclidean homographies. By decomposing the Euclidean homographies into separate translation and rotation components, reconstructed Euclidean information was obtained for the control development. A Lyapunovbased stability argument was used to design an adaptive update law to compensate for the fact that the reconstructed translation signal was scaled by an unknown depth parameter. The impact that the development in this paper makes is that a new analytical approach has been developed using homography-based concepts to enable the position/orientation of a WMR subject to nonholonomic constraints to track a desired trajectory generated from a sequence of images, despite the lack of depth measurements. Our future efforts will target experimental demonstration of the developed controller and the development of analytical Lyapunov-based methods for WMR visual servo tracking using an off-board camera similar to the problem in [9] without the restriction that the camera by fixed perpendicular to the WMR plane of motion.

\section{REFERENCES}

[1] S. Baker and S. Nayar, "A Theory of Catadioptric Image Formation," Proc. of the ICCV, pp. 35-42, Jan. 1998.

[2] D. Burschka and G. Hager, "Vision-Based Control of Mobile Robots," Proc. of the IEEE International Conference on Robotics and Automation, 2001, pp. 1707-1713.

[3] F. Chaumette and E. Malis, "2 1/2 D Visual Servoing: A Possible Solution to Improve Image-based and Position-based Visual Servoings," Proc. of the IEEE International Conference on Robotics and Automation, 2000, pp. 630-635.

[4] F. Chaumette, E. Malis, and S. Boudet, "2D 1/2 Visual Servoing with Respect to a Planar Object," Proc. of the Workshop on New Trends in Image-Based Robot Servoing, 1997, pp. 45-52.

[5] J. Chen, A. Behal, D. Dawson, and Y. Fang, "2.5D Visual Servoing with a Fixed Camera," Proc. of the IEEE American Control Conf., to appear.

[6] P. I. Corke and S. A. Hutchinson, "A New Hybrid Image-Based Visual Servo Control Scheme," Proc. of the IEEE Conference on Decision and Control, 2000, pp. 2521-2527.

[7] A. K. Das, et al., "Real-Time Vision-Based Control of a Nonholonomic Mobile Robot," Proc. of the IEEE International Conference on Robotics and Automation, 2001, pp. 1714-1719.

[8] K. Deguchi, "Optimal Motion Control for Image-Based Visual Servoing by Decoupling Translation and Rotation," Proceedings of the Intl. Conf. on Intelligent Robots and Systems, 1998, pp. 705-711.

[9] W. E. Dixon, D. M. Dawson, E. Zergeroglu, and A. Behal, "Adaptive Tracking Control of a Wheeled Mobile Robot via an Uncalibrated Camera System," IEEE Transactions on Systems, Man, and Cybernetics -Part B: Cybernetics, 31(3), pp. 341-352, 2001 .

[10] W. E. Dixon, D. M. Dawson, E. Zergeroglu and A. Behal, Nonlinear Control of Wheeled Mobile Robots, Springer-Verlag London Limited, 2001

[11] Y. Fang, A. Behal, W. E. Dixon and D. M. Dawson, "Adaptive 2.5D Visual Servoing of Kinematically Redundant Robot Manipulators," Conference on Decision and Control, Las Vegas, NV, Dec. 2002, pp. 2860-2865.

[12] Y. Fang, D. M. Dawson, W. E. Dixon, and M. S. de Queiroz, "2.5D Visual Servoing of Wheeled Mobile Robots," Conference on Decision and Control, Las Vegas, NV, Dec. 2002, pp. 28662871.

13] Y. Fang, W. E. Dixon, D. M. Dawson, and J. Chen, "Robust 2.5D Visual Servoing for Robot Manipulators," Proc. of the
2003 IEEE American Control Conf., Denver, CO, June 2003, pp. 3311-3316.

[14] O. Faugeras, Three-Dimensional Computer Vision, The MIT Press, Cambridge Massachusetts, 2001.

[15] O. Faugeras and F. Lustman, "Motion and Structure From Motion in a Piecewise Planar Environment," International Journal of Pattern Recognition and Artificial Intelligence, 2(3), 1988, pp. 485-508.

[16] G. D. Hagar, D. J. Kriegman, A. S. Georghiades, and O. BenShahar, "Toward Domain-Independent Navigation: Dynamic Vision and Control," Proc. of the IEEE Conference on Decision and Control, 1998, pp. 3257-3262.

[17] M. Hebert, "3-D Vision for Outdoor Navigation by an Autonomous Vehicle," Proc. of the Image Understanding Workshop, Cambridge, UK, 1998.

[18] B. H. Kim, et al., "Localization of a Mobile Robot using Images of a Moving Target," Proc. of the IEEE International Conference on Robotics and Automation, 2001, pp. 253-258.

[19] D. J. Kriegman, E. Triendl, and T. O. Binford, "Stereo Vision Navigation in Buildings for Mobile Robots," IEEE Transactions on Robotics and Automation, 5(6), Dec. 1989, pp. 792-803.

[20] Y. Ma, J. Kosecka, and S. Sastry, "Vision Guided Navigation for Nonholonomic Mobile Robot," IEEE Trans. on robotics and Automation, 15(3), June 1999, pp. 521-536.

[21] R. Mahony and T. Hamel, "Visual Servoing Using Linear Features for Under-actuated Rigid Body Dynamics," Proc. of the IEEE/RJS International Conference on Intelligent Robots and Systems, 2001, pp. 1153-1158.

[22] E. Malis and F. Chaumette, "Theoretical Improvements in the Stability Analysis of a New Class of Model-Free Visual Servoing Methods," IEEE Transactions on Robotics and Automation, 18(2), April 2002, pp. 176-186.

[23] E. Malis and F. Chaumette, "2 1/2 D Visual Servoing with Respect to Unknown Objects Through a New Estimation Scheme of Camera Displacement," International Journal of Computer Vision, 37(1), June 2000, pp. 79-97.

[24] E. Malis, F. Chaumette, and S. Bodet, "2 1/2 D Visual Servoing," IEEE Transactions on Robotics and Automation, 15(2), April 1999, pp. 238-250.

[25] J. J. E. Slotine and W. Li, Applied Nonlinear Control, Prentice Hall, Inc: Englewood Cliff, NJ, 1991.

[26] K.-T. Song and J.-H. Huang, "Fast Optical Flow Estimation and Its Application to Real-time Obstacle Avoidance," Proc. of the IEEE International Conference on Robotics and Automation, 2001, pp. 2891-2896.

[27] M. W. Spong and M. Vidyasagar, Robot Dynamic and Control, John Wiley and Sons, Inc: New York, NY, 1989.

[28] C. E. Thorpe, M. Hebert, T. Kanade, and S. Shafer, "Vision and Navigation for the Carnegie-Mellon Navlab," IEEE Trans. Pattern Anal. Machine Intell., 10(3), May 1988, pp. 362-373.

[29] M. A. Turk, D. G. Morgenthaler, K. D. Gremban, and M. Marra, "VITS-A Vision System for Autonomous Land Vehicle Navigation," IEEE Trans. Pattern Anal. Machine Intell., 10(3), May 1988 , pp. $342-361$.

[30] H. Y. Wang, S. Itani, T. Fukao, and N. Adachi, "Image-Based Visual Adaptive Tracking Control of Nonholonomic Mobile Robots," Proc. of the IEEE/RJS International Conference on Intelligent Robots and Systems, 2001, pp. 1-6.

[31] A. M. Waxman, et al., "A Visual Navigation System for Autonomous Land Vehicles," IEEE Journal of Roboics Automation, RA-3(2), 1987, pp. 124-141.

[32] Z. Zhang and A. R. Hanson, "Scaled Euclidean 3D Reconstruction Based on Externally Uncalibrated Cameras," IEEE Symp. on Computer Vision, 1995, pp. 37-42.

[33] H. Zhang and J. P. Ostrowski, "Visual Servoing with Dynamics: Control of an Unmanned Blimp," Proc. of the IEEE International Conference on Robotics and Automation, 1999, pp. 618-623. 